\title{
SHEAR COAXIAL INJECTOR INSTABILITY MECHANISMS $94-2,04$
}

T. Kaltz, M. Glogowski, and M. M. Micci

Aerospace Engineering Deparment \&

Propulsion Engineering Research Center

The Pennsylvania State University

University Park, PA 16802

\section{SUMMARY/OVERVIEW:}

Although stable operating regimes for cryogenic coaxial injectors have been empirically determined, there is no knowledge of the spray characteristics corresponding to stable operation, or the physical processes which produce the atomization patterns that result in stable or unstable operation. The current engineering method for determining the stable operating regime of a cryogenic coaxial injector is the 'hydrogen temperature ramping' method, however there is no definitive knowledge of whether the hydrogen temperature influences the chamber stability by decreasing the injected gas velocity. by affecting a recirculation region at the base of the LOX post, or by changing the pressure drop across the injector, allowing chamber pressure oscillations to couple to the fuel feed system. Results for the injector response from a linearized lumped-element model are presented as a function of temperature and frequency. LDV measurements in the recess region at the base of the LOX post show reverse flow indicative of a recirculation region. Finally, Phase Doppler Particle Analyzer (PDPA) measurements of droplet size and velocity distributions are discussed for a coaxial injector element similar to the SSME preburner element operating with water and air at atmospheric pressure and liquid and gaseous nitrogen at 20 bars.

\section{TECHNICAL DISCUSSION:}

In the 'hydrogen temperarure ramping' test one or more injectors are placed in a combustion chamber and hot fired while the temperature of the gaseous hydrogen being injected is slowly reduced until a spontaneous instability occurs. The physical significance of the hydrogen temperature ramping technique comes from the atomization process occurring in coaxial injectors where the high velocity outer gaseous hydrogen flow strips droplets from the lower velocity inner liquid oxygen flow. Experiments at ONERA using water as the liquid oxygen simulant have shown that a higher relative velocity between the two flows, one gaseous and the other liquid, results in smaller droplets'. Lowering the gaseous hydrogen temperature increases its density, thus lowering its injection velocity relative to the liquid in order to maintain the same mass flow and therefore the same fuel-to-oxidizer ratio. Wanhainen et al have shown that $i t$ is not the hydrogen temperature itself causing the transition to instability but

\footnotetext{
'Vingert, L., "Coaxial Injector Spray Characterization for the Ariane 5 Vulcain Engine," 6th Annual Conference "Liquid Atomization and Spray Systems - Europe," July 4-6, 1990.
} 
the ratio of the gas to liquid injection velocities?2. From this one might infer that the instability arises from a decline in droplet buming efficiency because of an increase in the liquid oxygen drop sizes along with an extension in the length of the atomization zone. A primary purpose of the experiments is to identify what effect the velocity ratio has on atomization and pressure drop across the injector to provide a better understanding of this common stability rating technique.

Another proposed explanation for the emergence of unstable operation in the hydrogen temperature ramping test is that a recirculation region acting as a flameholder exists downstream of the LOX post tip. Below a minimum relative velocity between the liquid oxygen and gaseous hydrogen, the recirculation region becomes too weak to act as a flameholder and the combustion zone moves away from the injector face 10 a location where it can interact more strongly with the chamber acoustic modes. Liang and Schumann have examined this idea with an experimenta] and computational investigation of gaseous oxygen and hydrogen coaxial injectors ${ }^{3}$. They examined several injectors designed to produce recirculation regions of different sizes but found that all injectors tested showed the combustion region anchored to the base of the injector. Whether this conclusion applies to liquid oxygen and gaseous hydrogen coaxial injectors has yet to be experimentally proven.

Some previous experimental evidence has indicated that the spontaneous instability condition for a hydrogen/oxygen rocket was determined by the hydrogen pressure drop through the injector.s. Below a critical minimum value for the pressure drop, chamber oscillations evidently can couple to the propellant feed system, causing a combustion instability in the chamber. However, the value of this critical pressure drop was a function of the injector and chamber design.

The first nine months of the grant period were spent designing a liquid nitrogen cooled heat exchanger to lower the temperature of the injected gas, either hydrogen or helium, from ambient down to $80 \mathrm{~K}$. The design has been completed and construction has begun. The heat exchanger will be used for the final series of experiments to simulate the hydrogen temperature ramping test.

An injector with the same dimensions as the SSME preburner injector has been constructed. The SSME preburner injector was selected because the test facility is capable of providing the required gas and liquid mass flow

\footnotetext{
${ }^{2}$ Wanhainen, J.P., Parish, H.C. and Conrad, E.W., Effect of Propellant Injection Temperature on Screech in 20,000-Pound Hydrogen-Oxygen Rocket Engine, NASA TN D-3373, April 1966.

${ }^{3}$ Liang, P.-Y. and Schumann, M.D., "A Numerical Investigation of the Flame-Holding Mechanism Downstream of a Coaxial Injector Element, "Proceedings of the 24th JANNAF Combustion Meeting, CPIA Publication 476, Vol. 3, Oct. 5-9, 1987, pp. 599-610.

${ }^{4}$ Conrad, E.W., Bloomer, H.E., Wanhainen, J.P. and Vincent, D.W., Interim Summary of Liquid Rocket Acoustic-Mode-Instability Srudies at a Nominal Thrust of 20,000 Pounds, NASA TN D-4968, Dec. 1968.

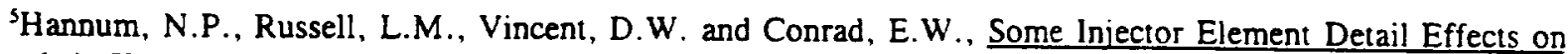
Screech in Hydrogen-Oxygen Rockets, NASA TM X-2982, Feb. 1974.
} 
rates for a full size injector and as large an injector as possible was desired to provide the possibility of optical access. The initial tests of the injector used air and water injecting into atmospheric ambient pressure. PDPA measurements were made of the droplet size and velocity as a function of axial and radial position. Both the magnitudes and the trends of the measured Sauter mean diameters and velocities agree well w'ith results obtained by Zaller and Klem ${ }^{6}$ using a similar injector and air and water flow rates. The main purpose for this limited series of tests was to confirm the operation of the PDPA system as well as the injector. The effects of varying the PDPA PMT voltage and of taking measurements through plexiglass windows of varying thickness were quantified. A windowed cold flow high pressure chamber has been designed, constructed and pressure tested to $9 \mathrm{MPa}$. As an initial step in the investigation of the atomization behavior of cryogenic coaxial injectors, liquid nitrogen was selected as an appropriate simulant to liquid oxygen based on their similar thermophysical properties. The fuel simulant for these first tests was gaseous nitrogen. The LN2/GN2 flow rates were computed from the nominal exit velocities of LOX/GH2 in the SSME fuel preburner injector. At a chamber pressure of 10 bar it was not possible to prevent cavitation at the lowest attainable supply temperature of the liquid nitrogen. Based on the vapor pressure of $\mathrm{LN}_{2}$ at that temperature, a chamber pressure of approximately 20 bar was required to avoid this phenomenon. Tests were pursued in which the pressure was maintained above 20 bar but yielded a dense spray that could not be measured satisfactorily by the PDPA. To obtain acceptable spray data it will be necessary to move further downstream in the spray and/or reduce the mass flow rate. These actions will be pursued in future tests.

The original injector design was modified in order to allow LDV access into the recess region. This was accomplished by inserting a pyrex tube concentrically around the LOX post, such that the fuel annulus was defined by a transparent boundary $0.04 \mathrm{in}$. thick. The inner diameter of the fuel annulus is 0.197 in. Gaseous nitrogen and water at flow rates of $1.8 \mathrm{gm} / \mathrm{s}$ and $1 \mathrm{gm} / \mathrm{s}$ respectively were used. From Figure 1 , it can be seen that recirculation does in fact exist within the recess region and the magnitudes of the axial velocities in this region appear consistent with the given flow conditions as does the trend on going from a high velocity gas to a low velocity liquid.

A simple model based on the Rayleigh criterion was constructed for the flow dynamics of several processes in the system to determine the effect of various design and operating parameters on combustion stability. The mechanisms included are the fuel flow through the injector, the vaporization of oxidizer in the chamber, and the flow of exhaust gases through the nozzle. Figures 2 and 3 show the effect of fuel temperature on the injector response for a chamber pressure of 21.4 bars and a gaseous fuel flow rate of $0.064 \mathrm{~kg} / \mathrm{s}$. Both graphs indicate that decreasing fuel temperature increases the response. At a frequency of $1 \mathrm{kHz}$ significant variations in response occur. Figure 2 indicates that the injector response is positive over a wide frequency range while Figure 3 shows that the injector response for certain frequencies can abruptly switch from stabilizing to destabilizing as the fuel

'Zaller, M., and Klem, M., "Shear Coaxial Injector Spray Characterization," Proceedings of the First International Symposium on Liquid Rocket Engine Combustion Instability, Jan. 18-20, 1993, University Park, PA. 
temperature is reduced. This supports the previous hypothesis that many low-frequency instabilities are due to the coupling of the feed system with the chamber acoustic modes. Results of this analysis will be compared to chamber high frequency pressure measurements. Measurements of the injector pressure drop as a function of the relative gas/liquid flow velocity and injector geometry are planned. The results of these measurements will be used as input to a stability model of the propellant feed system to determine the magnitude of the influence of the injector pressure drop on the combustion stability of the entire motor.

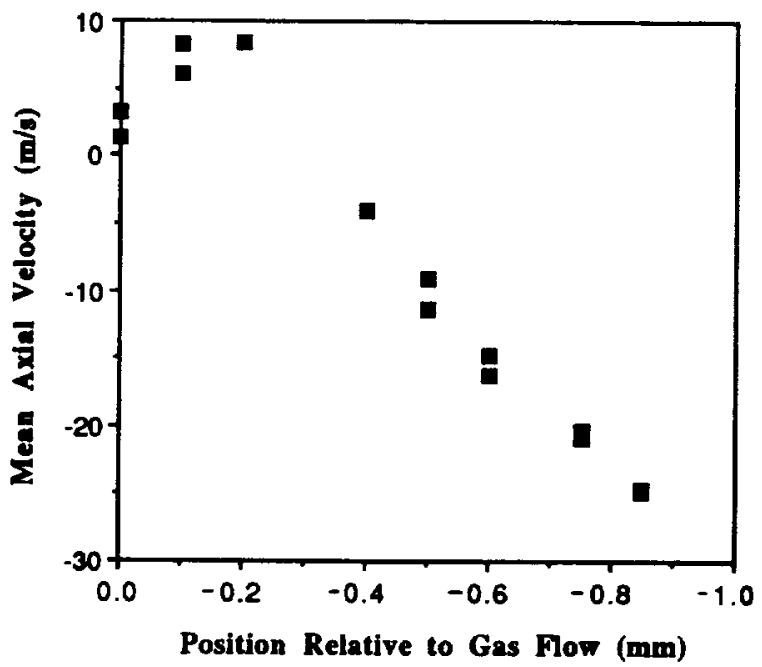

Fig. 1 LDV measurements of the mean axial velocity in the injector recess region showing reverse flow.

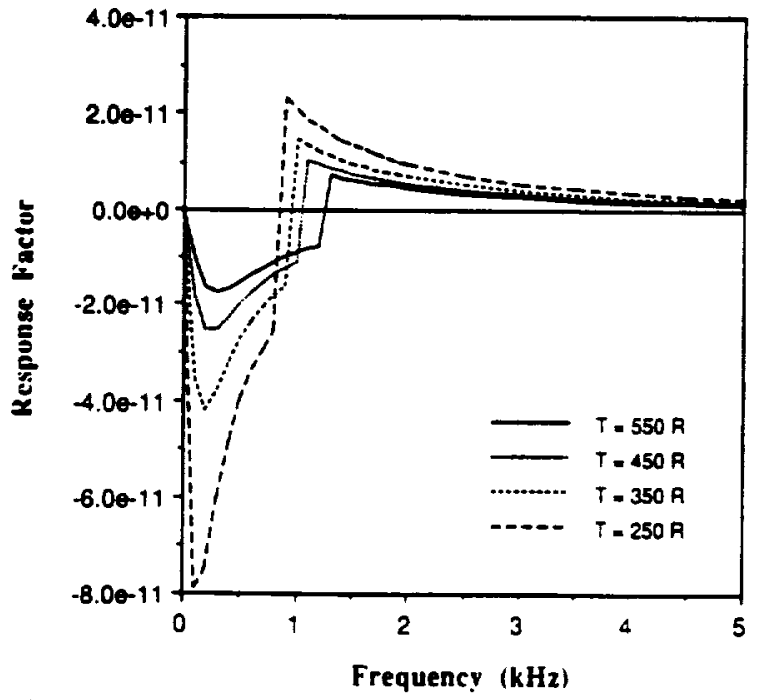

Fig. 2 Injector response as a function of frequency for several gaseous fuel temperatures.

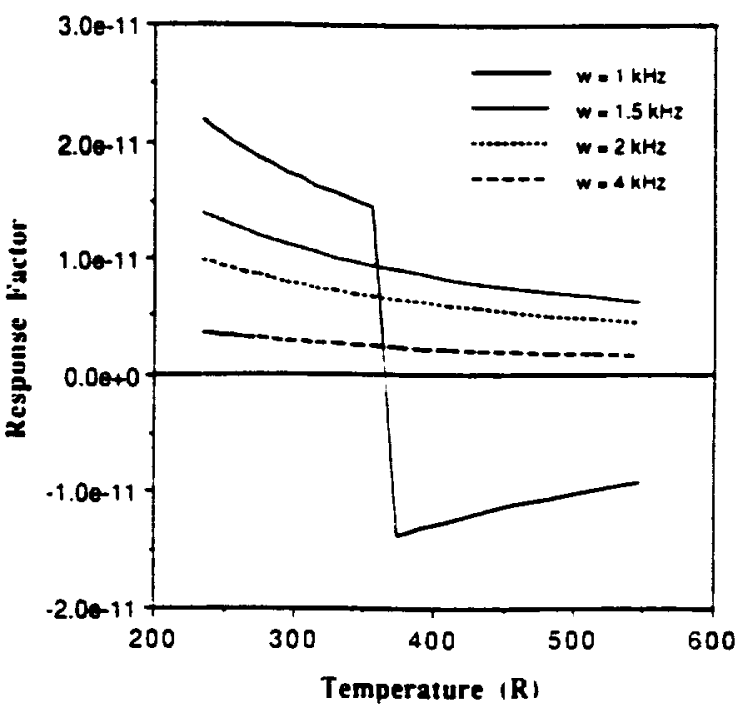

Fig. 3 Injector response as a function of gaseous fuel temperature for several frequencies. 